\title{
Multiple-Choice Questions in Preclinical and Clinical Phase Examination for Undergraduate Medical Program in a Malaysian Medical School: A 2-Year Analysis
}

Siti Khadijah Adam ( $\square$ sk.adam@upm.edu.my )

University Putra Malaysia https://orcid.org/0000-0003-2716-5705

Faridah Idris

Universiti Putra Malaysia

Puteri Shanaz Jahn Kassim

Universiti Putra Malaysia

Nor Fadhlina Zakaria

Universiti Putra Malaysia

Rafidah Hod

Universiti Putra Malaysia

\section{Research article}

Keywords: Item analysis, Multiple choice question, Difficulty index, Discrimination index, Distractor efficiency

Posted Date: July 7th, 2020

DOI: https://doi.org/10.21203/rs.3.rs-38264/v1

License: (c) (i) This work is licensed under a Creative Commons Attribution 4.0 International License.

Read Full License 
1 Multiple-choice questions in preclinical and clinical phase examination for undergraduate

2 medical program in a Malaysian medical school: A 2-year analysis

3 Siti Khadijah Adam $^{1,2^{*}}$, Faridah Idris ${ }^{1,3}$, Puteri Shanaz Jahn Kassim ${ }^{1,4}$, Nor Fadhlina Zakaria ${ }^{5}$,

4 Rafidah Hod ${ }^{1,2}$

$5{ }^{1}$ Medical Education Research and Innovation Unit, Faculty of Medicine and Health Sciences,

$6 \quad$ Universiti Putra Malaysia, Serdang, Selangor, Malaysia.

7 2Department of Human Anatomy, Faculty of Medicine and Health Sciences, Universiti Putra

8 Malaysia, Serdang, Selangor, Malaysia.

9 3Department of Pathology, Faculty of Medicine and Health Sciences, Universiti Putra Malaysia,

10 Serdang, Selangor, Malaysia.

11 4Department of Family Medicine, Faculty of Medicine and Health Sciences, Universiti Putra

12 Malaysia, Serdang, Selangor, Malaysia.

$13{ }^{5}$ Department of Medicine, Faculty of Medicine and Health Sciences, Universiti Putra Malaysia,

14 Serdang, Selangor, Malaysia.

15

16

17

18

19

20

21 
2 Medical Education Research and Innovation Unit, and

3 Department of Human Anatomy,

4 Faculty of Medicine and Health Sciences,

5 Universiti Putra Malaysia,

643400 Serdang,

7 Selangor Darul Ehsan,

8 Malaysia.

$9 \quad$ Tel: $+603-97692354$

10 Fax: $+603-97692341$

11 E-mail: sk.adam@upm.edu.my

12

13

14

15

16

17

18

19

20 


\section{Abstract}

Background: Multiple-choice questions (MCQs) are used in measuring the student's progress and post-examination analysis is usually done to guarantee the item's appropriateness for question banking. Item analysis is a simple and effective method to determine the quality of MCQs by using three parameters; difficulty or passing index (PI), discrimination index (DI) and distractor efficiency (DE).

Methods: This study analysed the MCQs in the preclinical and clinical examinations in Doctor of Medicine program of Universiti Putra Malaysia. Forty MCQs consisted of four options each in the preclinical examination and 80 MCQs with five options from the clinical examination paper in 2017 and 2018 were analysed and compared.

Results: The mean DI was similar in all examinations, except a significant reduction in 2018 clinical examination. From 2017 and 2018, preclinical MCQs showed an increment in the number of 'excellent' and 'good' items. However, clinical papers showed reduction in DI due to high number of 'poor' questions. Comparing both years, there was an increase in the number of items with no non-functioning distractors in both examinations. Among all, preclinical MCQs in 2018 showed the highest mean of DE as compared to the others.

Conclusion: Our findings suggested that item authors from preclinical phase showed an improvement in constructing good quality MCQs while clinical phase authors may need more training and continuous feedback. Higher number of options did not affect the level of difficulty of a question however the discrimination power and effectiveness of distractors might differ.

Keywords: Item analysis, Multiple choice question, Difficulty index, Discrimination index, Distractor efficiency 


\section{Background}

2 Doctor of Medicine (MD) curriculum in Universiti Putra Malaysia (UPM) consists of two phases;

3 pre-clinical and clinical phases which runs for 2 and 3 years, respectively. Students will sit for a summative assessment at the end of each phase. Students must pass the preclinical examination to proceed to clinical phase. In the fifth year, they need to sit and pass the clinical phase examination before awarded with the degree of medicine. In the written examination, various assessment tools are used such as MCQs, short answer questions and modified essay questions.

In any medical examinations, MCQ is one of the most important well-established written assessment tools widely used for its distinct advantage and ability to evaluate over a broad coverage of concepts in less time. The scoring is also objective and reliable [1]. The type A MCQ item (single best response) consists of a 'stem' or 'vignette', followed by a 'lead in' statement and several options. The correct answer in the list of options is called as a 'key' and the incorrect options are called 'distractors'.

The validity of an assessment refers to the evidence presented to support or to refute the meaning or interpretation assigned to the assessment data [2]. Validity, therefore, is a degree to which the test measures what it is supposed to measure. This includes test item analysis which

17 is usually done after the assessment has completed to determine the candidate responses to individual test items, the quality of those items as well as the overall assessment. Difficulty index or passing index $(\mathrm{PI})$, discrimination index (DI) and distractor efficiency (DE) of each item can be obtained from the analyses which reflect the quality of the test items. The PI of an item is commonly defined as the percentage of students who answered the item correctly. The DI, on the

22 other hand, is defined as the degree to which an item discriminates between students of high and 23 low achievement. The DE is used to assess the credibility of the distractors in an item, whether 24 they are able to distract students from selecting the right answer [3]. Any distractor that is selected 25 by less than $5 \%$ of the students is considered to be a non-functional distractor (NFD). The 
1 advantages of the analysis are to help identify faulty items, identify the lower performers and their

2 learning problems such as misconceptions as a guide for remedial actions to be done to students

3 and as importantly to increase educator's skills to construct a high quality of test items.

For this reason, we analysed the MCQs given in the preclinical and clinical phase

5

\section{Item analysis}

22 Post-validation was done automatically by item analysis using the OMR machine (Scantron 23 iNSIGHT 20 OMR scanner, Minnesota USA). The scores of all students in each examination 
1 paper were arranged in order of merit. The upper $27 \%$ students were considered as 'top' students

2 and lower $27 \%$ students as 'poor' students. Each item was analysed for difficulty and

3 discrimination indices according to Hassan \& Hod [4] and Abdul Rahim [5] :

4 (i) Difficulty index or Passing Index (PI), using the formula

$5 \quad \mathrm{PI}=(\mathrm{H}+\mathrm{L}) / \mathrm{N}$

6

7 (ii) Discrimination index (DI), using the formula

8

$\mathrm{DI}=(\mathrm{H}-\mathrm{L}) / \mathrm{A}$

9

10

$H=$ number of 'top' students answering the item correctly

11

$L=$ number of 'poor' students answering the item correctly

12

$\mathrm{N}=$ total number of students in the 'top' and 'poor' groups

13

$A=$ number of students in $27 \%$ of total students

14

15 The interpretation of PI and DI values are presented in Table 1.

16

17 (iii) Distractor efficiency (DE)

Non-functional distractor (NFD) is the option that was selected by less than $5 \%$ of students. Based on NFDs in an item, DE ranges from $0 \%$ to $100 \%$. If an item with four options contains three or two or one or nil NFDs, then DE would be $0,33.3 \%, 66.7 \%$ and 

NFDs, then DE would be $0,25 \%, 50 \%, 75 \%$ and $100 \%$, respectively.

3

$4 \quad$ Table 1 Interpretation of item analysis

\begin{tabular}{llll}
\hline Difficulty @ Passing Index (PI) & Values & Discrimination Index (DI) & Values \\
\hline Difficult & $\leq 0.3$ & Excellent & $\geq 0.35$ \\
Ideal & $0.31-0.59$ & Good & $0.25-0.34$ \\
Acceptable & $0.6-0.7$ & Acceptable & $0.21-0.24$ \\
Very Easy & $>0.7$ & Poor & $\leq 0.2$
\end{tabular}

5

6

${ }^{\star}$ Adapted from Ananthakrishnan [6]

\section{Results}

A total of 240 MCQs were analysed and the average PI and DI were determined. Overall, it was found that the difficulty level of the questions was similar in both preclinical and clinical phase examinations (Table 2). Interestingly, the values of average PI reduced in 2018, indicating a reduction of an increase of difficulty level of the questions for that particular year in both preclinical and clinical examinations. Nevertheless, the mean DI was similar in all examinations except for a low DI in the 2018 clinical phase examination.

For preclinical phase examination, the numbers of 'difficult' and 'very easy' items were similar in both years (Table 3). Half of the 40 MCQs were 'ideal' and 'acceptable'. Clinical phase examination in 2017 had 54\% 'ideal' and 'acceptable' items but the value reduced in 2018 to $34 \%$ due to the significant increase in the percentage of 'difficult' items in the year 2018. 
1 Table 2 Average of difficulty index and discrimination index of items in preclinical phase

2 examination $(n=40)$ and clinical phase examination $(n=80)$ in 2017 and 2018

\section{Mean \pm SD}

Preclinical Phase Examination Clinical Phase Examination

\begin{tabular}{lcccc} 
& 2017 & 2018 & 2017 & 2018 \\
\hline Difficulty @ Passing & $0.60 \pm 0.24$ & $0.55 \pm 0.23$ & $0.60 \pm 0.21$ & $0.56 \pm 0.28$ \\
Index (PI) & $($ Acceptable) & $($ Ideal) & (Acceptable) & (Ideal) \\
\hline Discrimination Index & $0.25 \pm 0.16$ & $0.31 \pm 0.16$ & $0.25 \pm 0.15$ & $0.20 \pm 0.11$ \\
(DI) & $($ Good $)$ & $($ Good $)$ & $($ Good) & (Poor)
\end{tabular}

4 Table 3 Summary of no of items in preclinical phase examination $(n=40)$ and clinical phase

5 examination $(n=80)$ in 2017 and 2018 based on difficulty index (PI)

\begin{tabular}{|c|c|c|c|c|}
\hline \multirow{3}{*}{$\begin{array}{l}\text { Difficulty @ } \\
\text { Passing Index } \\
(\mathrm{PI})\end{array}$} & \multicolumn{4}{|c|}{ Number of Items (\%) } \\
\hline & \multicolumn{2}{|c|}{ Preclinical Phase Examination } & \multicolumn{2}{|c|}{ Clinical Phase Examination } \\
\hline & 2017 & 2018 & 2017 & 2018 \\
\hline Difficult & $7(17.50)$ & $7(17.50)$ & $7(8.75)$ & $21(26.25)$ \\
\hline Ideal & $12(30.00)$ & $15(37.50)$ & $29(36.25)$ & $18(22.50)$ \\
\hline Acceptable & $8(20.00)$ & $5(12.50)$ & $14(17.50)$ & $9(11.25)$ \\
\hline Very Easy & $13(32.50)$ & $13(32.50)$ & $30(37.50)$ & $32(40.00)$ \\
\hline
\end{tabular}

6

7 There was an increment in the total percentages of 'excellent' and 'good' items in

8 preclinical phase examination in 2018 , from about $38 \%$ to $70 \%$ (Table 4 ). In clinical phase

9 examination of 2017, half of the questions were 'excellent' and 'good'. However, the percentage

10 reduced to $36 \%$ in 2018 due to the high percentage of 'poor' questions in the examination (53\%). 
1 Additionally, there were five questions with zero DI in the paper; one with PI equals to 1 and

2 another with $\mathrm{PI}$ equals to 0.

3

4 Table 4 Summary of number of items in preclinical phase examination $(n=40)$ and clinical phase

5 examination $(n=80)$ in 2017 and 2018 based on discrimination index (DI)

\begin{tabular}{|c|c|c|c|c|}
\hline \multirow{3}{*}{$\begin{array}{l}\text { Discrimination Index } \\
\text { (DI) }\end{array}$} & \multicolumn{4}{|c|}{ Number of Items (\%) } \\
\hline & \multicolumn{2}{|c|}{ Preclinical Phase Examination } & \multicolumn{2}{|c|}{ Clinical Phase Examination } \\
\hline & 2017 & 2018 & 2017 & 2018 \\
\hline Excellent & $11(27.50)$ & $15(37.50)$ & $23(28.75)$ & $6(7.50)$ \\
\hline Good & $4(10.00)$ & $13(32.50)$ & $17(21.25)$ & $23(28.75)$ \\
\hline Acceptable & $9(22.50)$ & $3(7.50)$ & $10(12.50)$ & $9(11.25)$ \\
\hline Poor & $16(40.00)$ & $9(22.50)$ & $30(37.50)$ & $42(52.50)$ \\
\hline
\end{tabular}

6

7 The total number of non-functioning distractors (NFD) was reduced in 2018 in both 8 examination phases (Table 5). Both examinations in 2018 showed an increase in the number of 9 items with no NFD as compared to the previous year. The number of items with no NFD in 10 preclinical phase examination is higher than clinical phase examination for both years. Similarly, 11 the overall mean DE was increased in 2018 with preclinical phase examination showing the 12 highest mean DE as compared to the rest of examinations. 
1 Table 5 Distractor efficiency (DE) of multiple-choice questions in preclinical phase examination

2 and clinical phase examination in 2017 and 2018

\begin{tabular}{|c|c|c|c|c|}
\hline \multirow[t]{4}{*}{ Parameter } & \multicolumn{4}{|c|}{ Number of Items (\%) } \\
\hline & \multirow{2}{*}{\multicolumn{2}{|c|}{$\begin{array}{c}\text { Preclinical Phase } \\
\text { Examination }\end{array}$}} & \multirow{2}{*}{\multicolumn{2}{|c|}{$\begin{array}{l}\text { Clinical Phase } \\
\text { Examination }\end{array}$}} \\
\hline & & & & \\
\hline & 2017 & 2018 & 2017 & 2018 \\
\hline Number of items & \multicolumn{2}{|c|}{40} & \multicolumn{2}{|c|}{80} \\
\hline Distractors & \multicolumn{2}{|c|}{120} & \multicolumn{2}{|c|}{320} \\
\hline Functioning distractors & $83(56.67)$ & $86(71.67)$ & 155 (48.44) & $177(55.31)$ \\
\hline Non-functioning distractors & $52(43.33)$ & $34(28.33)$ & $165(51.56)$ & $143(44.69)$ \\
\hline Items with no NFD (DE $=100 \%)$ & $9(22.50)$ & $13(32.50)$ & $3(3.75)$ & $8(10.00)$ \\
\hline Items with 1 NFD (DE $=66.67 \%)$ & $15(37.50)$ & $20(50.00)$ & $21(26.25)$ & $24(30.00)$ \\
\hline Items with 2 NFDs (DE = 33.33\%) & $11(27.50)$ & $7(17.50)$ & $28(35.00)$ & $32(40.00)$ \\
\hline Items with 3 NFDs (DE $=0$ ) & $5(12.50)$ & $0(0.00)$ & $24(30.00)$ & $9(11.25)$ \\
\hline Items with 4 NFDs $(D E=0)$ & - & - & $4(5.00)$ & $7(8.75)$ \\
\hline Overall mean DE & $56.67 \%$ & $71.67 \%$ & $48.44 \%$ & $55.31 \%$ \\
\hline
\end{tabular}

\section{Discussion}

5 The end of phase examination in UPM MD program is a high-stake summative assessment at the

6 end of preclinical and clinical phase. For preclinical phase examination, the results determine

7 whether the preclinical students are eligible to progress to clinical phase, while the final year

8 students need to pass the clinical phase examination to graduate. Therefore, valid assessment

9 tools are needed to measure students' knowledge, skills and attitude in the examination. One of

10 the tools used to test the 'knows' and 'knows how' in Miller's pyramid is with MCQ [7]. It is useful

11 in measuring factual recall, but it can also test higher order of thinking skills such as application, 
analysis, synthesis and evaluation of knowledge, which are important for medical graduates. Post-

2 validation of test items using item analysis of $\mathrm{PI}$, DI and DE is a simple, yet effective method to 3 assess the validity of the test. In the present study, we analysed the MCQs from both preclinical

4 and clinical phase examinations taken by two different cohorts of students. Each MCQ in 5 preclinical phase examination has four options while clinical phase examination has five options. Based on the findings, the mean PI in both examinations in both years were similar. This indicates that an increased number of options, five versus four options, does not have a significant impact on the difficulty level of the examination. This was supported by previous study by Schneid et al. [8] which found that there was no significant differences in the difficulty level among MCQs with three, four or five options. In contrary, Vegada et al. [9] found a slight decreased in the difficulty level when reducing the options from five to four, and the items became much easier when reducing the options to only three. They concluded that the items became easier with less number of options due to the increased probability of random guessing to select the correct answer.

Preclinical phase examination showed a consistent level of item difficulty for both years. Yet, half of the questions were 'very easy' and 'difficult'. These questions seem to be unsuitable to assess students in the high-stake examination as they were unable to discriminate between the good and the weak students. Hence, these questions should be revised, by changing either the vignette or the options. All difficult questions should be reviewed for its language and

$21 \mathrm{PI}$ as a percentage, which reflects the percentage of correct answers to the total responses [10-

22 12]. An item with PI percentage between $30 \%$ to $70 \%$ is considered as acceptable, i.e. not too easy and not too difficult [11, 12]. Studies by Sim \& Rasiah [10] and Rao et al. [11] showed a comparable mean PI similar to our present finding, ranging from 50 to $60 \%$. It is evident that with 
suggested that a continuous training and feedback should be given to the question authors so

2 that the number of 'difficult' and 'very easy' questions can be reduced in the future

3

4

The mean DI for preclinical phase examination in both years, and 2017 clinical phase examination ranging from 0.25 to 0.31 which were considered as 'good'. An earlier study had shown that a comparable mean of DI proved that the quality of questions has been consistent along the years [4]. Nevertheless, the mean DI in clinical phase examination reduced significantly in 2018. This may be due to the high number (66\%) of 'very easy' and 'difficult' questions in the examination. Consequently, about $53 \%$ of the questions were considered as 'poor' and were not able to discriminate between the good and weak students. A test item ideally should be able to pick out the 'good' students from the 'poor' ones, in which more 'good' students are able to answer the item as compared to the 'poor' students [5]. In the present study, some questions were found to have zero and negative DI. Zero DI means that the item was non-discriminating in which either all students were able to answer the item correctly, or an equal number 'good' and 'poor' students were able to answer correctly, or none of the 'good' and 'poor' students managed to correctly answer it. Negative DI indicates that more 'poor' students were able to answer the item correctly. In this study, one question was found to have zero DI and zero PI. These demonstrated an extremely low number of students who managed to answer it correctly and none of them were the 'good' and 'poor' students. We speculate the reasons for these were due to ambiguous framing of the questions, poor preparation of students or perhaps, wrong answer key was given $[10,13,14]$. One other question has zero $\mathrm{DI}$ and $\mathrm{PI}$ equals to 1 demonstrating that all students managed to answer it correctly, probably because it was too easy. Too difficult and too easy questions may contribute to the 'poor' questions based on the dome-shaped correlation between PI and DI $[12,15]$. These questions were not useful and may reduce the validity of the test, therefore should be eliminated. 
Preclinical phase examination in 2018 showed an increase in the number of 'excellent' and 'good' questions, and a decrease in 'poor' questions comparing to 2017 . This proves that the preclinical lecturers have shown considerable improvement in constructing MCQs through continuous training and feedback. In contrast, less than $8 \%$ of the questions in 2018 clinical phase examination were considered as 'excellent' and there was an increased number of 'poor' questions compared to 2017. Several possible reasons to this finding were identified. Some of the clinical lecturers were new and probably were unfamiliar with the test format, while some clinical lecturers had never attended any training on test item construction. There should be a thorough and several levels of vetting by peers, who are content experts and non-content experts is also needed before the questions are used in an examination. With this analysis, feedback should be given to all authors for them to reflect upon and revise their questions accordingly.

The number of NFDs also affects the discrimination power of an item [11]. From this study, more than $20 \%$ of the MCQs for preclinical phase had no NFDs in both years. In fact, there was one third of the 2018 preclinical phase MCQs with three functioning distractors (DE $=100 \%$ ). We identified that items with higher number of options in clinical phase examination tend to have higher NFDs in both years. Less than $10 \%$ of the MCQs for clinical phase examination had $100 \%$ DE. This shows that it was probably difficult for the authors to develop four equally plausible distractors. Preclinical phase examination in 2018 has no question with 0\% DE; and it showed the highest overall mean DE as compared to other examinations. Preclinical lecturers have shown a significant improvement in developing MCQs with plausible distractors and avoiding NFDs between the years. The findings suggested that reducing the number of options may increase the credible of distractors in an item, although it may reduce its difficulty level.

A meta-analysis by Rodriguez [16] found that having three options in an item is adequate. Even though the difficulty level is lowered, but it is more discriminating and more reliable. This is supported by a more recent study which found that questions with even as low as three options 
1 would still produce good reliability and less laborious to construct [17]. However, this means that

2 students will have high chance (only 1 in 3 ) of correctly answering the item with random guessing.

3 Royal \& Dorman [18] highlighted that 3-option and 4-option MCQs had similar psychometric

4 properties which means the former is equally effective as the latter. Therefore, the traditional

5 MCQs with 4 options shall be maintained as more research needs to be done to better understand

6 the effects of 3-option MCQs on guessing strategies and cut score determination decisions to

7 avoid any unintended consequential validity [19].

The present study highlights some interesting findings. First, an increased number of options does not affect the difficulty level of the questions, however, it does have significant effect on their discrimination power. Questions with higher number of options tend to have lower DI and

11 higher number of non-effective distractors. It is therefore suggested to standardize the number of 12 options to only 4, in both preclinical and clinical phases of examination. Second, question authors 13 from preclinical phase showed considerable improvement in constructing test items with plausible 14 distractors and ideal difficulty level as compared to the authors from clinical phase. Lack of 15 motivation and time constraints may be the possible challenges for the clinical authors to construct 16 good quality items [20]. Despite the availability of the faculty's guidelines for constructing 17 examination questions for reference, training and continuous follow-up and feedback to authors 18 are important to decrease items flaws and improve item-writing skills. Institutional support for 19 faculty development program is crucial to ensure reliable and valid assessment strategies 20 especially for high-stake examinations.

The roles of vetting committee in medical schools have been described in literatures to

22 evaluate the content, language and technical aspects of all questions [21, 22]. Vetting session

23 should be more thorough to ensure the validity of test items by removing any flaws and making 24 them as understandable and clear as possible [23]. Additionally, time as well as resources are 25 needed to develop the assessment blueprint to ensure all items are aligned with the learning 
1 objectives. A well-developed blueprint also corresponds with the depth of knowledge and level of

2 difficulty of each content area.

3 Several limitations should be noted in this study. First, this study was confined to one

4 educational setting, limiting its generalization. Any effort to infer the findings to other educational

5 settings needs to be done with caution. Second, several other parameters such as internal

6 consistency and correlation between PI and DI were not measured in this study. Lastly, some

7 variables such as author's previous training on writing MCQs and students' characteristics were

8 not controlled during the analysis which may affected the study findings.

10 Conclusion

11 The findings suggest standardizing the number of options to only four as it does not much affect

12 the difficulty level of the questions but will improve the degree of the items to discriminate between

13 high and low achievers. This will also ease the authors on writing MCQs with equally plausible

14 distractors. More trainings are required for the authors, especially from clinical phase to improve

15 the quality of the items as seen in preclinical authors. Feedback should be given to all authors

16 after analysis for them to reflect and make improvement. Good quality items shall be stored in the

17 question bank while the poor ones shall be discarded.

19 List of abbreviations

20 UPM: Universiti Putra Malaysia; MCQ: Multiple-choice questions; PI: Difficulty or passing index;

21 DI: Discrimination index; DE: Distractor efficiency; NFD: Non-functional distractor; SD: Standard

22 deviation 
2 Ethics approval and consent to participate

3 Not applicable.

4

5 Consent for publication

$6 \quad$ Not applicable.

7

8 Availability of data and materials

9 The data in the present study are available from the corresponding author on reasonable request.

11 Competing interest

12 The authors declare that they have no competing interests.

$14 \quad$ Funding

15 This study did not receive any specific grant from funding agencies.

17 Authors' contributions

18 SKA and $\mathrm{RH}$ contributed to the conceptualization and study design. Data analysis and

19 interpretation were performed by SKA, FI, PSJK and $\mathrm{RH}$. SKA drafted the manuscript. FI and NFZ

20 contributed substantially to its revision. All authors read and approved the final manuscript. 
2 The authors wish to thank the Dean and Deputy Dean (Academic) of Faculty of Medicine and

3 Health Sciences, UPM for their support and guidance. Also, appreciations to the staff in Academic

4 Unit of the faculty; Ms Siti Zuraida Shahardin, Ms Siti Nor Husaine Husin, Mr.Muhammad Hakimi

5 Bin Suhaimi and Mr. Mohd Esham Husain for their valuable assistance in data collection and 6 documentations.

7

8

9

10

11

12

13

14

15

16

17

18

19

20

21 
2 1. Kar S, Lakshminarayanan S, Mahalakshmy T. Basic principles of constructing multiple $3 \quad$ choice questions. Indian J Community Fam Med. 2015;1:65-9.

4 2. Messick S. Validity. In: Linn R, editor. Educational measurement. Washington, DC: $5 \quad$ American Council on Education; 1989. p. 13-103.

6 3. Linn R, Gronlund N. Measurement and Assessment in Teaching. 8th ed. New Jersey: $7 \quad$ Prentice Hall; 2000.

84 4. Hassan S, Hod R. Use of item analysis to improve the quality of single best answer multiple 9 choice question in summative assessment of undergraduate medical students in Malaysia. Educ Med J. 2017;9(3):33-43.

11 5. Abdul Rahim A. What those numbers mean: A guide to item analysis. Kota Bahru, Kelantan: KKMED Publications; 2010.

6. Ananthakrishnan N. Item analysis-validation and banking of MCQs. In: Sethuraman K, Kumar S, editors. Medical Education Principles and Practice. Pondicherry: JIPMER; 2000.

7. Miller G. The assessment of clinical skills/competence/performance. Acad Med. 1990;65(9):S63-7.

8. Schneid S, Armour C, Park Y, Yudkowsky R, Bordage G. Reducing the number of options on multiple-choice questions: Response time, psychometrics and standard setting. Med Educ. 2014;48(10):1020-7.

21 9. Vegada B, Shukla A, Khilnani A, Charan J, Desai C. Comparison between three option, four option and five option multiple choice question tests for quality parameters: $A$ randomized study. Indian J Pharmacol. 2016;48(5):571-5. 
1 10. Sim SM, Rasiah RI. Relationship between item difficulty and discrimination indices in true/false-type multiple choice questions of a para-clinical multidisciplinary paper. Ann Acad Med Singapore. 2006;35(2):67-71.

11. Rao C, Kishan Prasad H, Sajitha K, Permi H, Shetty J. Item analysis of multiple choice questions: Assessing an assessment tool in medical students. Int $\mathrm{J}$ Educ Psychol Res. 2016;2(4):201-4.

12. Kheyami D, Jaradat A, Al-Shibani T, Ali FA. Item analysis of multiple choice questions at the department of paediatrics, Arabian gulf university, Manama, Bahrain. Sultan Qaboos Univ Med J. 2018;18(1):e68-74.

13. Hassan S, Amin RM, Bt. Mohd Amin Rebuan H, Thwe Aung MM. Item analysis, reliability statistics and standard error of measurement to improve the quality and impact of multiple choice questions in undergraduate medical education in Faculty of Medicine at UniSZA. Malaysian J Public Heal Med. 2016;16(3):7-15.

14. Quaigrain K, Arhin AK. Using reliability and item analysis to evaluate a teacher-developed test in educational measurement and evaluation. Cogent Educ. 2017;4(1):1301013.

15. Mitra N, Nagaraja H, Ponnudurai G, Judson J. The levels of difficulty and discrimination indices in type a multiple choice questions of pre-clinical semester 1 multidisciplinary summative tests. Int eJournal Sci Med Educ. 2009;3(1):2-7.

16. Rodriguez MC. Three options are optimal for multiple-choice items: A meta-analysis of 80 years of research. Educ Meas Issues Pract. 2005;24(2):3-13.

17. Loudon C, Macias-Muñoz A. Item statistics derived from three option versions of multiplechoice questions are usually as robust as four- or five-option versions: Implications for exam design. Adv Physiol Educ. 2018;42(4):565-75. 
1 18. Royal K, Dorman D. Comparing item performance on three- versus four-option multiple choice questions in a veterinary toxicology course. Vet Sci. 2018;5(2):55.

3 19. Royal KD, Stockdale MR. The Impact of 3-Option Responses to Multiple-Choice Questions

4

5 on Guessing Strategies and Cut Score Determinations. J Adv Med Educ Prof. 2017;5(2):84-9.

20. Karthikeyan S, O'Connor E, Hu W. Barriers and facilitators to writing quality items for medical school assessments - A scoping review. BMC Med Educ. 2019;19(1):123.

21. Gopalakrishnan S, Udayshankar PM. Question vetting: The process to ensure quality in assessment of medical students. J Clin Diagnostic Res. 2014;8(9):XM01-XM03.

22. Hassan S, Simbak N, Mohd Yussof H. Structured vetting procedure of examination questions in medical education in Faculty of Medicine at Universiti Sultan Zainal Abidin Malaysia. Malaysian J Public Heal Med. 2016;16(3):29-37.

23. Wadi M. Question vetting: theory and practice. Educ Med J. 2012;4(1):e1-4. 